\title{
Dynamics of aroma-active volatiles in miso prepared from lizardfish meat and soy during fermentation: a comparative analysis
}

\author{
Anupam Giri, Toshiaki Ohshima* \\ Department of Food Science and Technology, TokyoUniversity of Marine Science and Technology, Tokyo, Japan
}

Email address:

ag2002dr@yahoo.co.in (A. Giri), tohshima@kaiyodai.ac.jp (T. Ohshima)

\section{To cite this article:}

Anupam Giri,Toshiaki Ohshima. Dynamics of Aroma-Active Volatiles in Miso Prepared from Lizardfish Meat and Soy during Fermentation: A Comparative Analysis. International Journal of Nutrition and Food Sciences. Vol. 1, No. 1, 2012, pp. 1-12.

doi: $10.11648 /$ j.jinfs.20120101.11

\begin{abstract}
The evolution of aroma-active compounds during the maturation of lizardfish meat and soybean miso was quantified and characterized using the purge-and-trap method for volatile isolation. The results revealed that miso prepared from lizardfish meat and soybeans is the result of alcoholic fermentation rather than acid fermentation. The miso prepared from soybeans matured earlier (60-90 days) than that prepared from lizardfish meat (135 days). The constancy in the volatile lipid-oxidation products, including certain aldehydes and ketones, indicated the oxidative stability of both miso products throughout the fermentation period. The present findings indicated several compounds responsible for miso aroma, including 2-methylpropanal, 3-methylbutanal, 3-methyl-1-butanol, ethyl isobutyrate, 2-methylethyl butanoate, 3-methylethyl butanoate, ethyl decanoate, 2,3-butanedione, dimethyl trisulfide, methional, and 2-methyl butanoic acid. The formation of aldehyde can be attributed to the decomposition of hydroperoxides and peroxyl radicals, which are supposed to be initial products of oxidized fat. The volatile ketones were most likely the products of lipid and/or amino acid degradation also could possibly be produced from secondary degradation reactions involving diverse substances from the lipid during fermentation and/or may be derived from the Maillard reaction. Formation of the major volatiles in miso products were suggested as a combined effect of fungal metabolism of amino acids, sugars, and lipids, as well as the Maillard reaction during the fermentation period. The major difference between miso prepared from lizardfish meat and that from soybeans was the relative abundance of those odor-active compounds that finally characterize the products. Substrate specificmetabolic capacity of A. oryzae and the Maillard reaction were presumed to determine the flavor profile of the end product of miso.
\end{abstract}

Keywords: Volatiles, Aroma-Active Compounds, Olfactometry, Fish Miso, Soy Miso

\section{Introduction}

Fish miso, a promising fermented fish meat paste made from different trash fishes, was previously investigated for its taste components and nutritional status [1,2], as well as for the headspace volatiles of a fully matured product [3]; however, the dynamics of headspace volatiles throughout the fermentation period has not yet been investigated. Miso is a typical example of a solid-state fermented (SSF) product by nature. Traditional fermentation such as that to make Japanese koji, Indonesian tempeh, and French blue cheese are typical examples of this technique. SSF is useful for the production of flavor compounds [4,5]. Feron \& Bonnarme [6] reviewed the microbial production of several food flavors through the SSF processes. Numerous microorganisms are capable of synthesizing potentially valuable flavor compounds and enzymes used in flavor manufacturing, such as Neurospora spp. [7], Zygosaccharomyces rouxii [8], and Aspergillus spp. [9] using pregelatinized rice, miso, and cellulose fibers, respectively. Compounds such as acetaldehyde, ethanol, ethyl acetate, ethyl isobutyrate, isobutyl acetate, isoamyl acetate, and ethyl-3-hexanoate were identified in the headspace of the cultures. The production of flavor compounds in dairy products, such as butyric acid, lactic acid, and diacetyl, in mixed cultures of Lactobacillus acidophilus and Pediococcus pentosaceus growing on a semisolid maize-based culture has been reported [10]. It is known that several methylketones such as 2-undecanone, 2-nonanone, and 2-heptanone are produced on a commercial scale by SSF from Aspergillus niger using coconut fat as a substrate with a yield of $40 \%$ [11]. However, as a newer 
product, very little information is available on the volatile profiles and evolution of aroma-active compounds in fish miso during fermentation. A clear understanding of the dynamics of aroma-active volatiles in miso during fermentation is believed to be important for process manipulation and investigation for improving flavor profiles.

The objectives of the present study were to follow the evolution of the aroma-active volatile compounds in miso prepared from lizardfish meat and soybeans during the fermenting process to obtain a more comprehensive understanding of which type of substances are responsible for its flavor. Attempts were also made to elucidate the mechanisms involved in the production of miso aroma-active volatiles.

\section{Materials and Methods}

\subsection{Materials and Chemicals}

Lizardfish (Saurida wanieso) with a mean body weight of $985 \pm 84 \mathrm{~g}$ and a fork length of $43.4 \pm 0.6 \mathrm{~cm}$ were caught by trawl nets offshore of Nagasaki prefecture. The fish were stored at $-50^{\circ} \mathrm{C}$ for a month until their use in miso production. All the volatile standards used for identification and other analysis purposes were of GC-analytical grade and purchased from Tokyo Chemical Industry Co., Ltd. (Tokyo, Japan).

\subsection{Preparation of Fermented Fish and Soy miso}

The fish were beheaded, gutted, and washed; the skin and bones were removed using a model NF2 deboning machine (Bibun Kikai Seisakusyo Co. Ltd., Fukuyama, Japan) equipped with a drum with perforations of $4 \mathrm{~mm}$ in diameter. After grinding each fish separately using a model M-22 grinder (Nantsune Tekko, Osaka, Japan), the ground meat was put into an aluminum-coated heat-stable polyvinylchloride pouch, vacuum-sealed, and then steamed at $90^{\circ} \mathrm{C}$ for $1 \mathrm{~h}$. Portions were then filter pressed at $2 \mathrm{MPa}$ to achieve a moisture content of $50-55 \%$ using a model KS-1 filter press (Komagata Kikai Seisakusho, Tokyo, Japan). The resulting dehydrated meat was used in the preparation of fish miso. Meanwhile, one of the major ingredients, koji, was prepared using Koshihikari rice (Oryza sativaproduced in Nigata prefecture, Japan) soaked in fresh water for $12 \mathrm{~h}$ at room temperature and subsequently steamed at $90^{\circ} \mathrm{C}$ for $1 \mathrm{~h}$. After cooling at room temperature to $35^{\circ} \mathrm{C}$, the rice was inoculated with koji mold (M1 mold, pure strain of A. oryzae; Nihon Jozo Kogyo, Tokyo, Japan) and incubated at $35^{\circ} \mathrm{C}$ for $48 \mathrm{~h}$, producing the malt rice used as koji.

Fish meat, koji, and salt were mixed using a grinder at the ratio of 5:5:1 by wet weight. Approximately $3 \mathrm{~kg}$ of fish paste was packed into a 5 -L plastic container and fermented between $25^{\circ} \mathrm{C}$ and $30^{\circ} \mathrm{C}$ for 365 days. The contents of each container were mixed thoroughly once a month. The prepared material is called "fish miso" (i.e., fermented fish paste).

Soybean miso was prepared using steamed soybeans
(Ootsubu tsurumusume, produced in Hokkaido prefecture, Japan), koji (as prepared for fish miso) as a starter, and salt. Soybeans were previously soaked in fresh water for $12 \mathrm{~h}$ and steamed at $90^{\circ} \mathrm{C}$ for $1 \mathrm{~h}$, after which the same procedure as that for fish miso was followed. The products were sampled at $0,15,30,60,90,135,180,270$, and 365 days for analysis.

\subsection{Isolation of Volatiles using Tenax TA trap}

Samples of $3 \mathrm{~g}$ with $0.01 \mathrm{~mL}$ of 1,000 ppm 2,4,6-trimethyl pyridine as an internal standard were placed in a $500-\mathrm{mL}$ dual-necked round-bottom glass flask and placed over a hot water bath maintained at $75^{\circ} \mathrm{C}$. High-purity nitrogen gas was passed through the smaller neck of the flask at a flow rate of $100 \mathrm{~mL} / \mathrm{min}$. Headspace volatiles emanating from the sample were trapped for 30 min on a fritted-glass absorption trap (4 mm I.D., $6 \mathrm{~mm}$ O.D. and 4.5 inch long; Supelco, Bellefonte, Pennsylvania, USA) filled with $175 \mathrm{mg}$ of $60 / 88 \mathrm{mesh}$ Tenax TA (Supelco) fitted to a bigger neck using a connector. A dry nitrogen purge was carried out for $10 \mathrm{~min}$ at a flow rate of $50 \mathrm{~mL} / \mathrm{min}$ in a direction opposite to the isolation to remove the residual moisture. The Tenax TA trap was preconditioned at $300^{\circ} \mathrm{C}$ for $30 \mathrm{~min}$ with nitrogen gas at 50 $\mathrm{mL} / \mathrm{min}$ using a Model 10 tube conditioner (Dynatherm Analytical Instruments Inc. Kelton, Pennsylvania, USA) before each sampling.

\subsection{Conditions of Gas Chromatography}

A dry-purged tube with isolated volatiles was placed onto a Model ACEM 900-FF/EPC automated thermal desorption system (CDS Analytical, Inc., Oxford, Pennsylvania, USA). Volatiles trapped in the dry-purged Tenax TA tube were desorbed for $3 \mathrm{~min}$ at $300^{\circ} \mathrm{C}$, and the volatiles were injected onto a capillary column in a narrow-band plug through a short path transfer line $\left(0.5 \mathrm{~m}\right.$ inert column kept at $\left.250^{\circ} \mathrm{C}\right)$. Analysis of volatiles were carried out on a Shimadzu model 14A gas chromatograph (Kyoto, Japan) equipped with a Supelcowax ${ }^{\mathrm{TM}}-10$ fused silica capillary polar column $(0.32$ $\mathrm{mm}$ i.d. $\times 60 \mathrm{~m}, 0.25 \mu \mathrm{m}$ in film thickness, Supelco). High-purity helium was used as a carrier gas at a flow rate of $1.5 \mathrm{~mL} / \mathrm{min}$ with a column inlet pressure of $3 \mathrm{~kg} / \mathrm{cm}^{2}$. The column temperature was held at $40^{\circ} \mathrm{C}$ for $3 \mathrm{~min}$ and subsequently programmed to $200^{\circ} \mathrm{C}$ at a rate of $3^{\circ} \mathrm{C} / \mathrm{min}$. Injector and detector temperatures were set at $250^{\circ} \mathrm{C}$.

\subsection{Conditions of GC-MS}

Analysis of the mass spectra of volatile compounds was carried out on a Hewlett Packard 5890 Series II gas chromatograph (Supelcowax ${ }^{\mathrm{TM}}-10$ fused silica capillary polar column: $0.25 \mathrm{~mm}$ i.d. $\times 60 \mathrm{~m}, 0.25 \mu \mathrm{m}$ in film thickness) equipped with a Tekmar 7000 headspace auto-sampler (Cincinnati, Ohio, USA) and an ion source from Automass (JEOL, Japan). The ionization energy, scan range, and scan rate applied for the analysis were $70 \mathrm{eV}, 40-350 \mathrm{~m} / \mathrm{z}$, and $500 \mathrm{~m} / \mathrm{s}$, respectively. The column temperature was initially held at $40^{\circ} \mathrm{C}$ for $3 \mathrm{~min}$ and subsequently programmed to $200^{\circ} \mathrm{C}$ at a rate of $3^{\circ} \mathrm{C} / \mathrm{min}$. 


\subsection{Identification and Quantification of Volatile Com- pounds}

The volatile compounds were identified by comparing the mass spectra, linear retention index (LRI), and retention times with those obtained using authentic standards. Kovats retention indices (RIs) were determined for both gas chromatography - flame ionization detection - olfactometry (GC-FID-O) and gas chromatography-mass spectrometry (GC-MS) using a series of $n$-alkanes $\left(\mathrm{C}_{5}-\mathrm{C}_{24}\right)$ and compared with those reported previously in the literature. The major volatile compounds were quantified using standard curves obtained from each compound from at least 5 different concentrations in methanol. The levels of the volatile compounds were normalized by 2,4,6-trimethyl pyridine equivalents (assuming that all response factors to the FID were 1). The methanolic solution of internal standard 2,4,6-trimethyl pyridine $(0.01 \mathrm{~mL}$ of $1,000 \mathrm{ppm})$ was introduced to the 3-g of sample in the flask just before the subsequent volatile isolation process. The odor activity values (OAVs) and odor notes described in the text were based on our previous findings [3].

\subsection{Measurement of Soluble Protein and Soluble sugar}

For the measurement of soluble protein and sugar, $1 \mathrm{~g}$ of sample was diluted in $10 \mathrm{~mL}$ of distilled water and centrifuged at $3,000 \times g$ for $15 \mathrm{~min}$ at $4{ }^{\circ} \mathrm{C}$. The supernatant was collected and added to $5 \mathrm{~mL}$ of $0.44 \mathrm{M} \mathrm{Na}_{2} \mathrm{CO}_{3}$ and $1 \mathrm{~mL}$ of
3 -fold diluted Folin-Ciocalteu reagent. The resulting solution was incubated for $30 \mathrm{~min}$ at $30^{\circ} \mathrm{C}$, and absorbance was read at $660 \mathrm{~nm}$. L-Tyrosine was used for expressing the amount of soluble protein.

Soluble sugars were determined by incubating $0.5 \mathrm{~mL}$ of the solution extract with $100 \mu \mathrm{L} \mathrm{3,5-dinitrosalicylic} \mathrm{acid}$ (DNS) reagent and heating in boiling water for $10 \mathrm{~min}$. The reducing groups catalyzed/reduced DNS, and the development of color was monitored spectrophotometrically at 540 $\mathrm{nm}$ after cooling in ice for $5 \mathrm{~min}$. The amount of soluble sugar was expressed as D-glucose equivalent.

\section{Results and Discussion}

\subsection{Quantification and Analysis of Evolution of Head- space Volatiles of Miso Prepared from Lizard-fish Meat and Soybean during Fermentation}

A total of 104 headspacevolatile compounds detected by GC-FID using Tenax TA traps from both lizardfish meat and soybean miso were identified using authentic compounds, mass spectra, and LRI with authentic standards. Most of the compounds were consistently quantified using standard curves and authentic standards. The calculated LRI and changes in the volatile concentration of miso prepared from lizardfish meat and soybeans during fermentation are presented in Table 1. The trends of volatile classes throughout the fermentation period were compared between the types of miso and are presented in Fig.1.
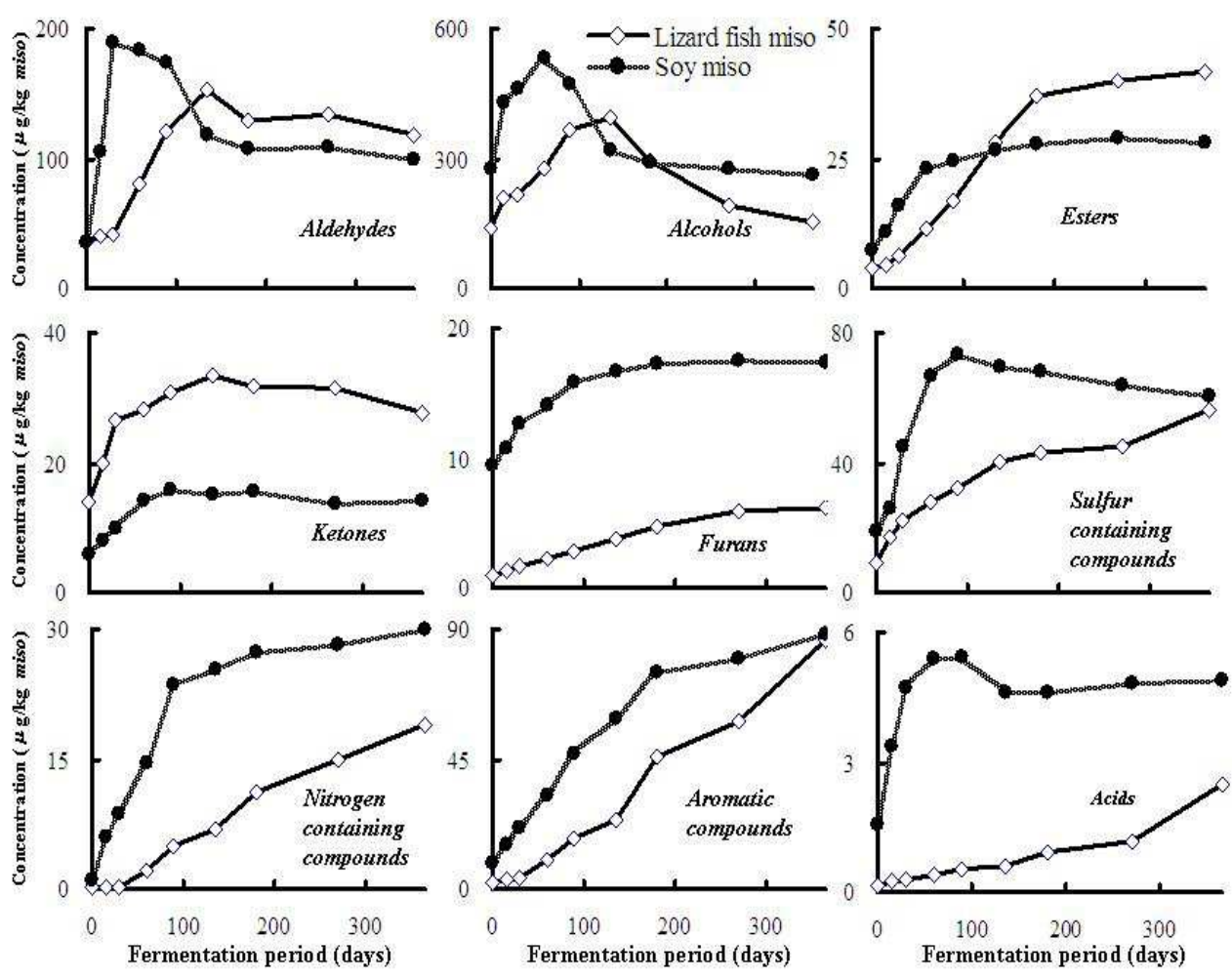

Figure 1. Changes in volatile classes during the fermentation period of fish miso prepared from lizard fish meat and soy bean. 
Ialite $\mathrm{I}$

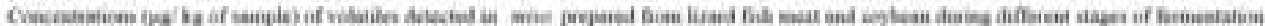

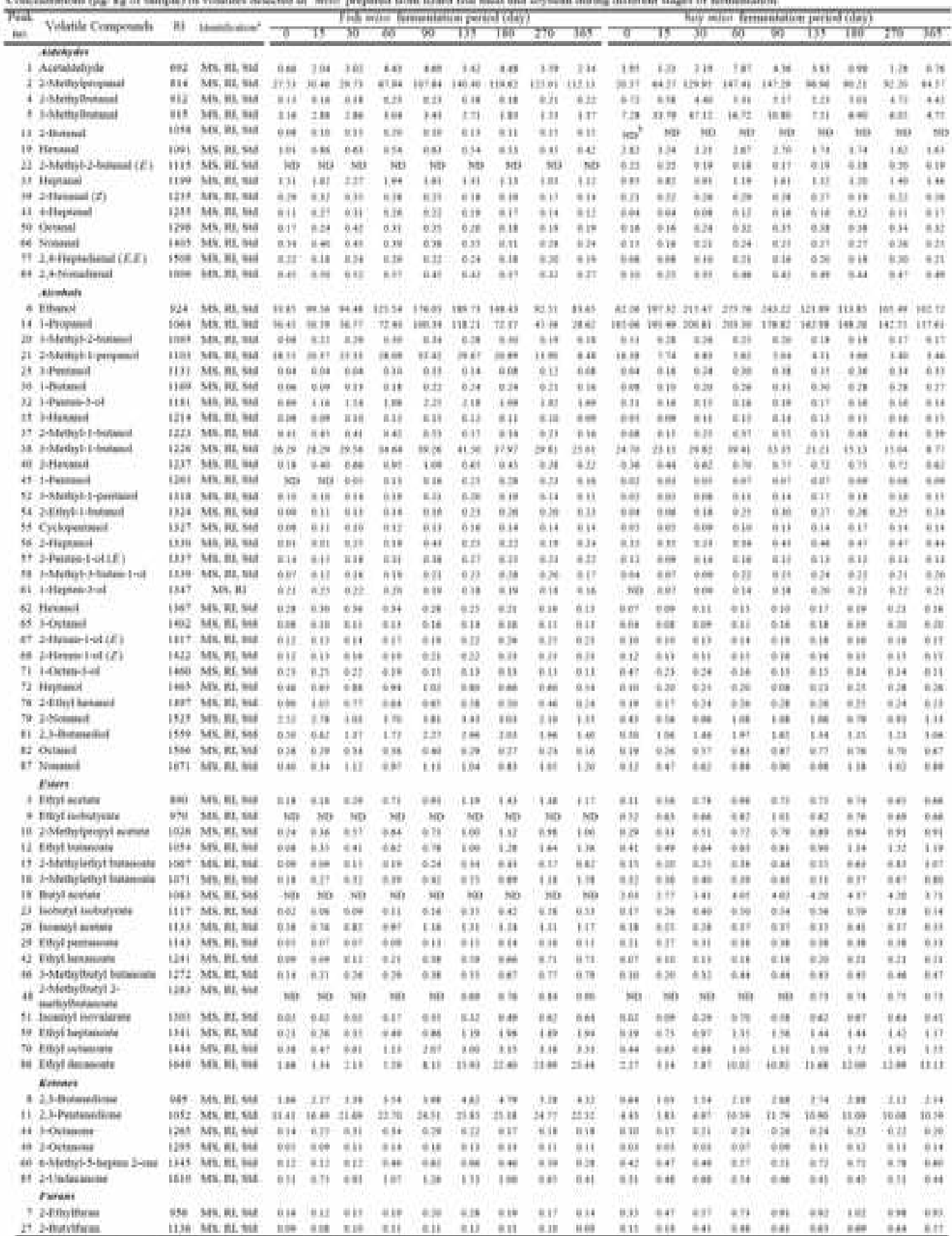

Most of the volatile compounds found were common in both the types of miso; however, the evolution of volatile compounds and their compositions differed. The volatile aldehyde class includes 13 compounds, while 12 were found to be common for both miso types. Comparing the trend of total volatile aldehydes with the progress of fermentation, it can be concluded that aldehydes were relatively higher in miso prepared from soybeans than in that prepared from 
lizardfish meat. The results also revealed that the total aldehyde content in soybean miso reached a maximum from 30 to 60 days of fermentation and then decreased gradually until the end of the 365-day fermentation period; however, the aldehyde content in miso prepared from lizardfish meat increased up to 135 days and then decreased gradually until the end of the process. The composition of volatile aldehydes clearly indicated that Strecker aldehydes, including 2-methylpropanal, 2-methylbutanal, and 3-methylbutanal, were major contributors of volatile aldehydes for both types of miso; however, aldehydes from lipid oxidation, including hexanal, heptanal, 2-hexenal (Z), 2,4-heptadienal, and 2,4-nonadienal, were found in small quantities and remained nearly unchanged until the end of fermentation period. This result suggested that oxidative stability was maintained throughout the fermentation period for both types of miso.

For both types of miso, the volatile alcohol class contributed to the major volatiles. Comparative studies revealed that total volatile alcohol content was higher for soybean miso, for which an initial rapid increase was observed early (15 days), reached its maximum during 60 days of fermentation, and gradually decreased at 90 days onwards. In contrast, the total volatile alcohol content in miso prepared from lizardfish meat gradually increased until 135 days of fermentation. Quantitative analysis revealed that ethanol, 1-propanol, 2-methylpropanol, and 3-methylbutanol made up a major portion of the volatile alcohol class for both types of miso. Among those, the trend of ethanol content in both miso types indicated the occurrence of alcoholic fermentation during the early stages of fermentation. In the fish miso, the process of alcoholic fermentation was relatively slow and reached its maximum during 135 days of fermentation, whereas it took only 60 days for soy miso. This is most likely because of the substrate specificity of A. oryzae to the fermenting condition in miso. The number of volatile alcohols - particularly branched-chain alcohols - was small, and most showed an increasing trend in the early stages of fermentation and a gradually decreasing pattern in the later stage of maturation.

The acetates of higher alcohols and the ethyl esters of fatty acids are perhaps the most desirable compounds in food products to enhance the aroma of the finished products. Seventeen volatile esters were detected in both types of miso. The total ester content was initially higher in soybean miso than in lizardfish meat miso; however, during the advanced stages of maturation (90 days forward) the content of total volatile esters increased rapidly in miso prepared from lizardfish meat until the end of the fermentation period ( 365 days). Among the volatile esters, butyl acetate, ethyl heptanoate, ethyl octanoate, and ethyl decanoate contributed the most in soybean miso. However, volatile esters of matured miso prepared from lizardfish meat were dominated by ethyl acetate, ethyl butanoate, isoamyl acetate, ethyl heptanoate, ethyl octanoate, and ethyl decanoate. Most of the volatile esters detected increased throughout the fermentation period.The total volatile ketone content increased in the early stages of fermentation. The content reached its maximum during 90 days and 135 days for soybean and lizardfish meat miso, respectively. Later, the total ketone gradually decreased. Volatile ketones in both types of miso were dominated by 2,3-butanedione and 2,3-pentanedione. The ketones from fungal metabolism and/or $\beta$-oxidation, namely 2-octanone and 2-undecanone, were detected in small quantities for both types of miso. Both the volatile ketones remained unchanged or increased slightly throughout the fermentation period, suggesting the oxidative stability of the product.

The total content of volatile furan and its derivatives gradually increased throughout the fermentation period regardless of miso type; however, a relatively higher content of furan derivatives was detected in soybean miso. 2-Furaldehyde and furfuryl alcohol were found to be the major contributors of this volatile class. Both volatile compounds increased throughout the fermentation period, particularly in fish meat miso, where a rapid increase was observed during the advanced stages of fermentation.

Sulfur-containing compounds were initially higher in soybean than in lizardfish meat miso and rapidly increased throughout 90 days of fermentation period. Subsequently, a gradually decreasing trend was observed from 90 days until the end of fermentation period at 365 days. In contrast, the total sulfur-containing compounds of fish miso gradually increased throughout the fermentation period. Among the sulfur-containing compounds, 3-(methylthio)propanal (methional), 3-(methylthio)propanol (methionol), 2-ethoxy thiazole, and dimethyl disulfide were detected in higher quantities in both types of miso. After an initial increase was observed for dimethyl disulfide and in the advanced stages of fermentation, it gradually decreased. In contrast, other sulfur-containing compounds, including methional, methionol, and 2-ethoxy thiazole, gradually increased throughout the fermentation period regardless of miso type.

Volatiles of nitrogen-containing compounds were detected in small quantities for both types of miso. Volatile pyrazines were detected mostly during the advanced stages of fermentation, suggesting their formation during the later stages of maturation; however, the 2-acetyl pyrrole content was relatively higher in soybean miso. In addition, 2-acetyl pyrrole gradually increased throughout the fermentation period.

A large number of volatile aromatic compounds were detected in both types of miso. Most of those increased throughout the fermentation period regardless of miso type; however, a rapid increase after 90 days of fermentation was observed in the fish miso. Among the volatile aromatic compounds, 4-ethyl guaiacol, 4-ethyl phenol, phenylethyl alcohol, 2-phenylethyl acetate, and benzaldehyde were detected as major volatiles belonging to this group in both miso products.

The total volatile acid content was relatively higher in soybean miso, in which it gradually increased until 90 days of fermentation. The volatile acid content was initially low for fish miso; however, the contents increased in the final stages of fermentation. Volatile acids detected from both 
miso products were 2-methyl propanoic acid, 2-methyl 3-methyl butanoic acid was predominant. butanoic acid, and 3-methyl butanoic acid, of which

\begin{tabular}{|c|c|c|c|c|c|c|c|c|c|c|c|c|c|c|c|c|c|c|c|c|}
\hline \multirow{2}{*}{ peil VolateComodi } & \multirow{2}{*}{ N } & \multirow{2}{*}{ Whinat } & \multicolumn{9}{|c|}{ 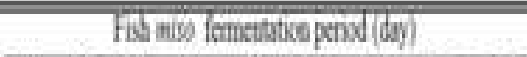 } & \multicolumn{9}{|c|}{ 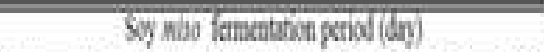 } \\
\hline & & & T) & 75 & 30 & 6 & 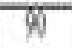 & 735 & 710 & 80 & 76 & 0 & 75 & W1 & (7) & 7) & 135 & Ti & 37 & 35 \\
\hline it Wentian & 124 & Ma, Ri, sid & (14) & 49 & the & bit & 611 & (1): & III & it & 177 & atis & 14: & Li: & 114 & is & 140 & 1.4 & 194 & 14 \\
\hline 7) Hinklibla & 114 & Mis & ait & as & aj & 4 & oit & 04 & 04 & as & in & \$4 & It & 24 & 19 & 1it & III & H & at & 40. \\
\hline 71 Matyling & 1910 & MERIAS & $d a$ & dis & 013 & Wit & oit & dit: & as: & da1 & is & 14 & if & 14 & 17 & 14t: & $m$ & 213 & 30 & wi) \\
\hline W lutulinitul & 10 & SWH & do & $d y$ & tal & wit & in & Iil & 1e & jiI & 17 & 111 & iL & 613 & iti & 14 & IM & ith & III & (i) \\
\hline \multicolumn{21}{|c|}{ 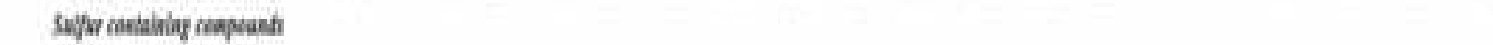 } \\
\hline if Dhatydiullik & Hil & M, 21,34 & art. & ask & 19. & 65 & be & $d x$ & ds & 64 & bذ & 4 & 21 & $w$ & $m$ & [1] & IF & 1111 & $d A$ & 101 \\
\hline 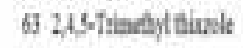 & 190 & M, 21, st & dit & oil & dit & on & 00 & ofi & an & Q6: & by & 611 & bit & on? & os & at & is & in & in & 16 \\
\hline 4 Denchinails & 1): & M, 14.4 & 60 & ait & ais & ix & 10 & bili & of & on & ais & 13 & ait & on & in & ait & bia & ant & ast & is \\
\hline 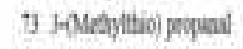 & 1170 & ME, Z1L,SH & (16) & wit : & 194 & thin & 81 & nis & MH & Ifis & 144 & 4it & ty & $H w$ & 46 & tis & 1615 & $\operatorname{tin}$ & 197 & tot \\
\hline 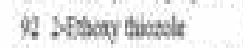 & wi & M, 14,54 & 610 & m & 07 & as: & to & or & 14 & 114 & 11t & 10 & 12 & 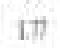 & 27 & 24 & 27 & 27 & in & $30 \mathrm{t}$ \\
\hline 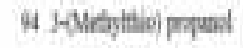 & tids & She & 10 & 苗 & uis & 24 & is & 44 & it & wi & int & 64 & 111 & 211 & (4) & 49 & ili & 14 & (4) & 47 \\
\hline \multicolumn{21}{|c|}{ 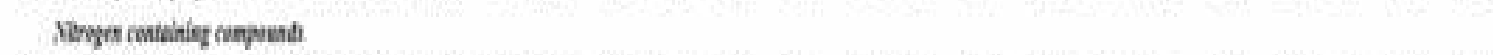 } \\
\hline 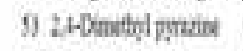 & 1nit & MSRLSH & abi & ath & Hat & bis & III & 191 & (4i) & in & III & aili & aif & I.t. & ja & 617 & 4II & ill & yy & $y$ \\
\hline of Trmithy paints & HIH & Minisy & 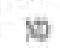 & 8 & sp & 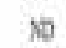 & ae & 617 & tit & 14: & 14 & SD & 9 & 10 & 8 & 10 & 14) & 111 & 14 & 137 \\
\hline 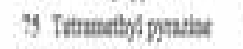 & Iith & Wus & N & 9 & $\mathrm{NO}$ & M & AH & $0 n$ & a! & 64 & 19 & SD & NP & No & $\$$ & an: & Q11 & त & in & Hi \\
\hline low Lhadphade & (क) & MG A, & dii & ail & dit & 2hi & $2 n$ & iti & in & $11 \mathrm{~s}$ & tiil & 10 & ba & fis & inil & Hit & $11 \mathrm{ti}$ & Woil & $x+4$ & Di1 \\
\hline \multicolumn{21}{|l|}{ Anutir caypuah } \\
\hline 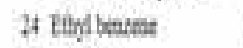 & HM & MS, 21, 네 & 011 & ai & 04 & bit & aib & 64 & ay & 64 & int & $M$ & dy & 02 & 190 & dit & $0 x$ & dit & ont & tiv \\
\hline if p-twes & $111 \mathrm{M}$ & $\mathrm{M}, 21, \mathrm{M}$ & ait & att & OB & ati & ait & (1i) & dit & det & at: & का & 曾 & 60 & 44 & al & bit & 614 & 614 & iit \\
\hline 9 on xylat & 1101 & M, R, 14 & 011 & oy & all & 111 & att & 0 & a11 & obs & 024 & oil & 017 & ont & (1) & (1) & 94 & ox & 64 & 100 \\
\hline 4 chand & wo & Min, 84 & 011 & 641 & ull & the & a11 & oil & th: & 64 & i11 & $b 4$ & 04 & 691 & 10 & 111 & 䊁 & on & $0 \mathrm{H}$ & toit \\
\hline 15 Ropl berus & 1311 & 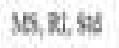 & ast & 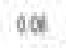 & tht? & bat? & 04 & tht & the & abt & int & ia) & 00 & 64 & 냉 & tef & do4 & 614 & 106 & toit \\
\hline it polyon & $11^{4}$ & MEI & $\mathrm{N}$ & 19 & 10 & 10 & (4) & $\mathrm{s}$ & 111 & 1411 & Hili & So & 40 & we & iD & 6 & क & 114 & 114 & Whit \\
\hline W Beraldyta & $\mid 144$ & MEL, & 幛 & ath & Dit & tit & at & tis & 44 & (1) & ow & on & (b) & 12 & ic & 14 & itt & 14 & if & 17 \\
\hline 8 L thithowati & W & Sin: 4 & 10 & $d a$ & (b) & 04 & an & 12 & 13 & III & ait & (11) & in & 64 & Ii & iff & in & 17 & 144 & IIII \\
\hline 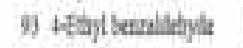 & $17 i$ & Mis, यू & tiii & 100 & thi! & 1018 & at & 6is & 14t & 64 & dy & this & ats & dabs & an & tis & dit & at & ail & (1) \\
\hline of flyphoylutith & $1 \mathrm{~W}$ & AS, $11,5 \mathrm{~B}$ & 60 & ath & 밴 & $6 x$ : & 16 & in & $2 \pi$ & 14) & 14 & bia & [11 & an & $\lim$ & 44 & 14 & 319 & 24 & 104 \\
\hline 9. 2 haylatyiste & III & MS, 제, 에 & 64 & at & 611) & (1) & 24 & Iit & (4) & 10 & in & on & 07 & 14 & III & Iit & itt & ita & is & 14 \\
\hline of poumal & (bi) & BS, 21 & $\omega$ & 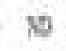 & No & $\mathrm{w}$ & 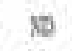 & ta & ali & at & 011 & 6at & bat & $6 \mathrm{at}$ & (a) & 46 & all & ant & byt & 411 \\
\hline of lanof boud & 119) & 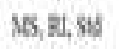 & ait & 014 & tal & 647 & a! & tit & det: & 64 & 144 & 044 & of & d9 & ni & tht & oit & it) & in & 111 \\
\hline w Prylebthod & DII & MB, 11,54 & on & on & 111 & 13 & $2 \pi$ & in & III & III & 167 & in & ati & IH & 111 & 24 & bii & 64 & \pm 4 & 19 \\
\hline 101 Padsl & $x^{4}$ & MS RI S4 & ajs & at & (1)! & tits & aj! & 6it & (14) & bit & as & bi: & 10.4 & 097 & 4 & ti1 & ail. & we & an & แ⿻ \\
\hline 10: tivgl frowal & 3il & MERISH & odd & od & od & (a) & ges & os & \$1! & oit & ad & 60 & oui & obs & at & ar & 011 & ail & 014 & (1) \\
\hline 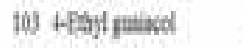 & 246 & MULW & at & ail & 04 & wi & in & 44: & 1117 & $19 y$ & 매영 & ith & का & 11. & 116 & xy & Hil & 47 & jth & Hai \\
\hline 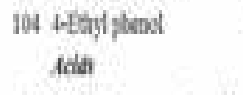 & wa & MS a , s & dit & dit? & th & tL & III & 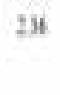 & jH & 14 & 164 & ot & at & 115 & 13 & i11 & itit & in & tit & tist \\
\hline 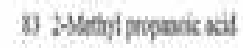 & 1958 & MS, RL,SII & 0 녀 & a1? & bit & 614 & 041 & 㛃 & 14 & 64) & бท & (1) & 041 & dy & U1) & 04 & bit & 榦 & oH & 请 \\
\hline 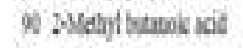 & 140 & 4. 14 & $\infty$ & $\nabla$ & 10 & bot & dil & Oif & dis & dis & thil & oil & iil & 64 & in & of & ail & b1 & 63 & 61 \\
\hline 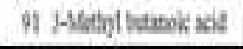 & (tai & Ma, at, st & 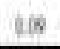 & a11 & till & ith & 语 & 베가 & itis & 14 & in & $11 i$ & iti & +31 & thit & 497 & in & 14 & bit & Iis \\
\hline
\end{tabular}

\subsection{Hypothetical Pathways for the Enzymatic Activity leading to Volatile Formation in Solid-state Fer- mented Miso prepared from Soybean and Lizard-fish Meat}

Miso flavor is a combined effect of a large number of aroma-active volatile compounds that are produced during fermentation. The presence of compounds that are precursors of the flavoring compounds, as well as suitable medium conditions, are necessary. In our previous studies $[1,2]$, biochemical analysis of miso prepared from both soybeans and fish meat revealed that both comprise a large number of substrates that are similar in composition or have similar properties; however, the major difference between them is the difference in raw materials and/or a different metabolic capacity of the enzymes derived through koji fermentation by A. oryzae. Our investigation on the enzyme activity of koji during fermentation indicated that enzyme activity in 
koji is high, especially protease and amylase activity. Our trials on the koji enzymes on lizardfish meat and soybean substrate clearly indicated that they can efficiently hydrolyze protein and carbohydrate substrates from fish origin (Fig.2), although the comparative studies indicated a sig- nificantly higher rate of enzyme activity in soybean substrate. Based on enzymatic activity, substrate specificity, and catabolic pathways of amino acids, sugars and lipids, the hypothetical mechanism of volatile production for both kinds of miso is explained here (Fig.3).
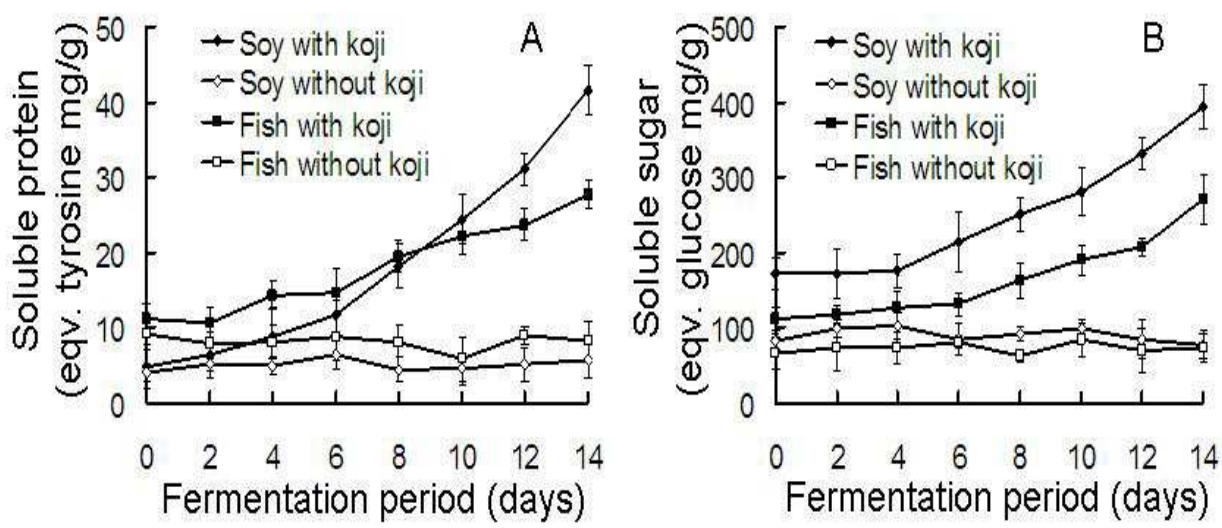

Figure 2. Effect of koji enzymes on the formation of soluble protein (A) and soluble sugar (B) from lizard fish meat and soy bean.
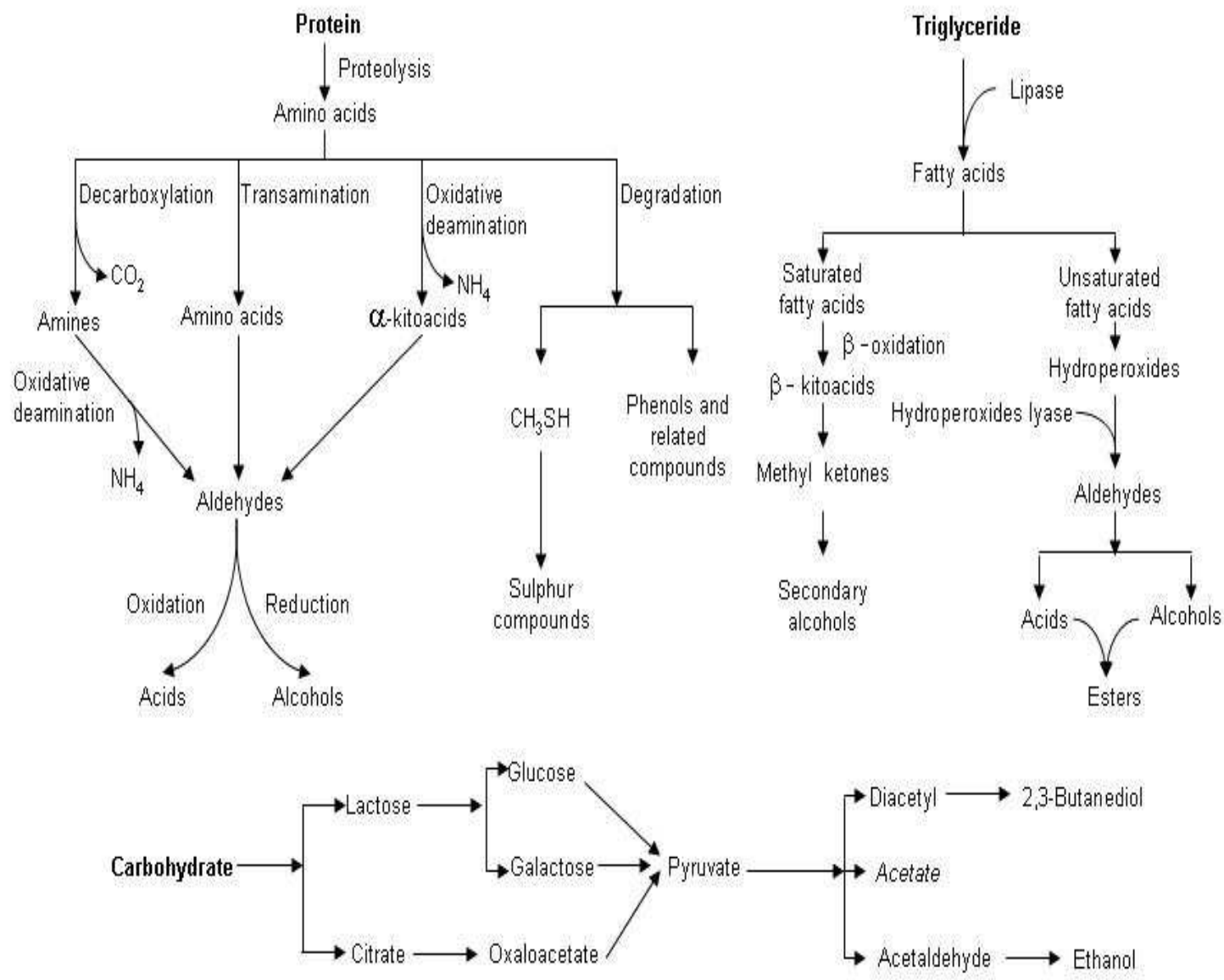

Figure 3. Hypothetical pathways of lipids, proteins and carbohydrates metabolism to different volatile compounds in miso prepared from lizard fish meat and soy bean.

\subsubsection{Amino Acids and Their Conversion Products}

Proteins play an important role in fish miso manufacturing. They not only raise the nutritional value but also con- tribute to the development of flavor by producing amino acids. For the development of an acceptable fish miso flavor, it is necessary to balance the breakdown of proteins into 
small peptides and amino acids. These products of proteolysis are known to contribute to the flavor or act as precursors of other flavor components during the formation of miso flavor. The major aromatic compounds in fermented miso products are generally produced as a result of catabolism of aromatic amino acids, beginning with a transamination step that produces indolepyruvate, phenylpyruvate, and p-hydroxyphenylpyruvate from tryptophan, phenylalanine, and tyrosine, respectively [12]. The authors also showed that some of these products were likely to be formed by non-enzymatic processes because spontaneous chemical degradation of the tyrosine intermediate, $\mathrm{p}$-hydroxyp henyl pyruvic acid, produced p-hydroxy pheny lacetic acid, p-hydroxy propionic acid, and p-hydroxy benzaldehyde, while the chemical degradation of the phenylalanine intermediate, phenylpyruvic acid, resulted in the production of phenylacetic acid, benzoic acid, phenylethanol, phenylpropionic acid, and benzaldehyde.

The miso volatiles contained several sulfur-containing compounds such as methional, dimethyl disulfide, dimethyltrisulfide, 2-ethoxy thiazole, and methionol, each of which contributes to the aroma of miso. They originate either from raw materials or during the fermentation process from free peptidic and proteinic sulfur amino acids, as well as the glutathione pool in fish tissue [13]. Hypothetical pathways of those sulfur-containing compounds in miso products are presented in Fig.4 as suggested earlier by Landaud, Helinck, \& Bonnarme [14] in several fermented products. Transamination is the first step in the degradation of amino acids such as methionine, leading to the formation of $\alpha$-keto acids ( $\alpha$-KA), which finally leads to the formation of several sulfur-containing volatile compounds [15]. In the present study, 3-(methylthio)propanal (methional), the Strecker aldehyde of methionine, was detected in both miso samples. The production of methional was reported as a process mediated by amino transferase and $\alpha-\mathrm{KA}$ decarboxylase activities [16]. 3-(Methylthio)propanol detected in miso samples has been reported to be produced by the 4-methylthio-2-oxobutyric acid, a transamination product of methionine, which yields methional, followed by a reduction step. Methionol might also contribute boiled potato flavor to the finished products. The sulfurs in heterocyclic sulfur compounds such as thiazoles may be derived from amino acids (cysteine, cystine, and methionine) or from thiamine (vitamin B1) [17]..

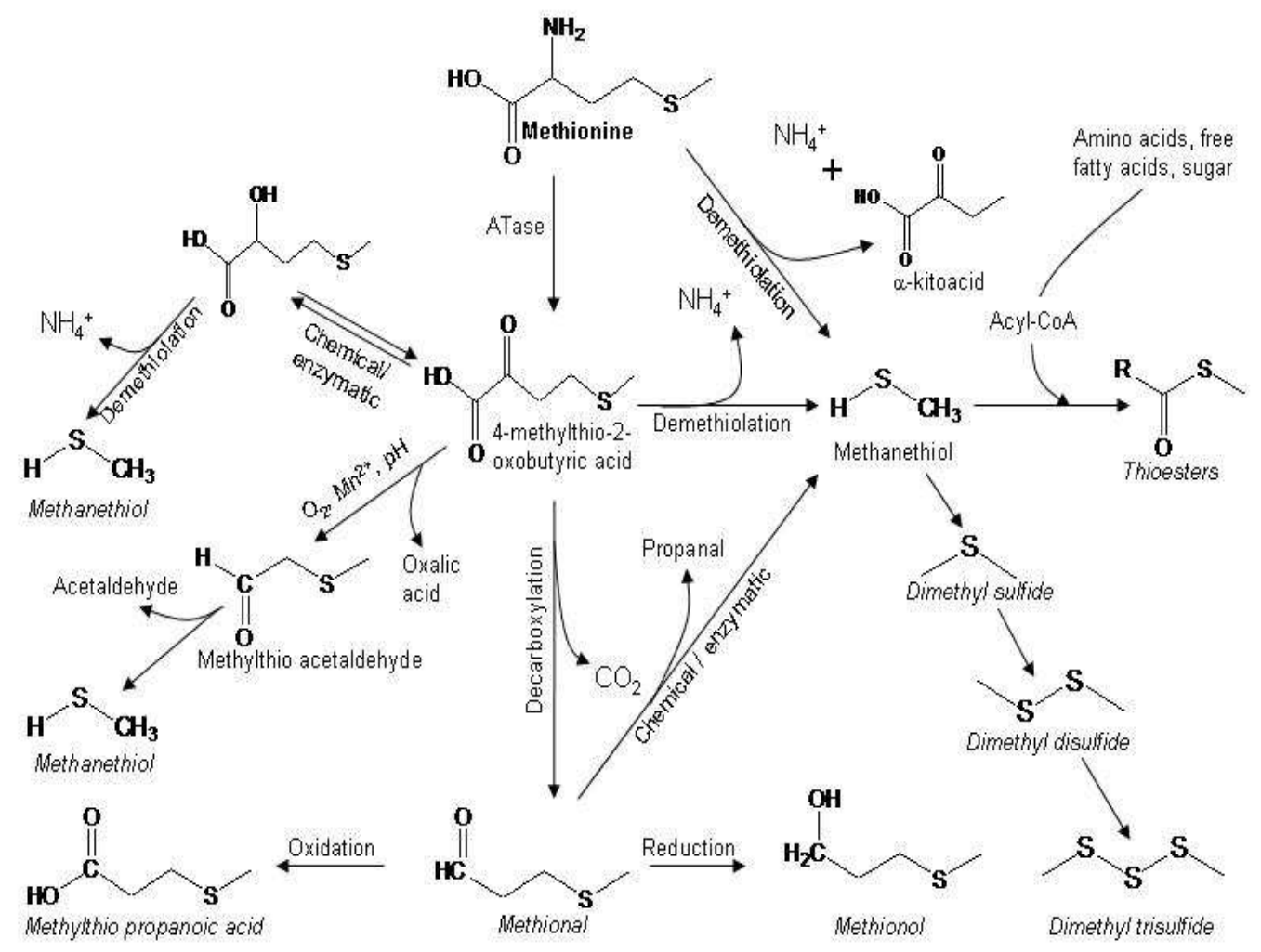

Figure 4. Hypothetical pathways of methionine catabolism to different volatile sulfur compounds in miso prepared from lizard fish meat and soy bean.

In the present study, the major part of the aldehydes detected for both types of miso was contributed by 2-methylpropanal, 2-methylbutanal, and 3-methylbutanal. In the regular catabolic pathway of amino acids, leucine is converted to $\alpha$-ketoisocaproic acid (4-methyl-2- oxopenta- noic acid) by deamination. $\alpha$-Ketoisocaproic acid is finally converted to acetoacetate and acetyl-CoA. The latter compound can enter the tricarboxylic acid cycle.As an alternative to the major catabolic route, $\alpha$-ketoisocaproic acid can be used as the starting molecule for the generation of the 
branched aldehyde, 3-methylbutanal

In some cases, they can be derived by Streckerdegradation of amino acids. For example, 2-methylbutanal may be derived from isoleucine [18]. In fish miso, the breakdown of intact proteins into peptides and free amino acids is a complex process that involves endogenous enzymes and koji proteinases and peptidases. Moreover, conversion of liberated amino acids into Strecker aldehydes and other flavor and aroma compounds might be the rate-limiting step in the development of aged flavors. The mechanism for the formation of aldehydes was demonstrated to form 2-methylpropanal, 2-methylbutanal, and 3-methylbutanal by way of a biosynthetic enzymatic/microbiologic pathway using Saccharomyces cerevisiae, another important koji-forming mold similar to A. oryzae [19]. The aldehyde formation might increase from the biosynthesis of valine, leucine, and isoleucine. Volatile acids can be produced as a result of either lipolysis or from amino acid metabolism (valine deamination) [20].

\subsubsection{Sugars and their Conversion Products}

The major sugars present in soybean are sucrose, raffinose, and stachyose, whereas soybean and fish miso contained a higher amount of glucose, arabinose, mannose, and galactose [1]. Several free sugar alcohols, including glycerol, erythritol, mannitol, and inositol, were also detected in fish and soybean miso. These sugars are generally fermentable by most lactic acid bacteria, bifidobacteria, fungi, and yeasts, and their metabolites are reported to act as precursors of several aroma-active compounds in various fermented products $[21,22]$. Many fungi, including A. oryzae, have been found to be capable of producing $\beta$-glucosidases, one of the major relevant enzymes for the production of food flavors during SSF $[23,24,25]$. $\beta$-Glucosidase showed good stability and tolerance to low $\mathrm{pH}$; therefore, it has potential applications in processing different fermented products for aroma and flavor enrichment through enzymatic hydrolysis of glucoside precursors. The glucosidases were also reported to act as slow-release aroma compounds [26]. Volatile flavors evaporate during storage, leading to a decrease in the correct concentration when consumed, but if they are present in a bound, non-volatile form, they can be liberated when heated, thus optimizing the organoleptic characteristics of the consumed product.

Sugar fermentation using yeasts such as S. cerevisiae, S. ellipsoideus, and a mixed culture of Aspergillus spp. yields alcohol; however, alcohol may be formed by decomposition of the secondary hydroperoxide of fatty acids [17]. Branched-chain alcohols detected in miso samples including 1-penten-3-ol, 2-methyl-1-butanol, 3-methyl-1-butanol, 3-methyl-1-pentanol, 2-ethyl-1-butanol, 2-penten-1-ol (E), and 1-octen-3-ol were reported to be produced during yeast fermentation from 2 different sources: carbohydrates by the Embden-Meyerhof pathway (EMP) and amino acids through the Ehrlich pathway. The Ehrlich pathway begins with the enzyme-catalyzed decarboxylation of branched-chain 2-oxo acids to the corresponding aldehydes.
Subsequently, the aldehyde is reduced to the corresponding fusel alcohol by an alcohol dehydrogenase. The branched-chain 2-oxo acid substrates for the Ehrlich pathway can be produced by the deamination of L-leucine, L-isoleucine, or L-valine. Growth of A. oryzae with any of these 3 amino acids as the nitrogen source might result in the accumulation of the corresponding fusel alcohol. Alternatively, branched-chain 2-oxo acids may be synthesized de novo from carbohydrates as intermediates of branched-chain amino acid synthesis. The conversion of branched-chain oxo acids into their respective aldehydes and alcohols through the Ehrlich pathway resembles the fermentative metabolism of pyruvate, which yields ethanol and carbon dioxide. In both cases, the decarboxylation of a 2-oxo acid is followed by the reduction of the resulting aldehyde [27,28]. Evidence of the formation of several branched-chain alcohols, including 3-methylbutanol and 1-octen-3-ol by several molds of Aspergillus, including A. oryzae, was reported from a $60 \%$ moistened wheat meal medium [29].

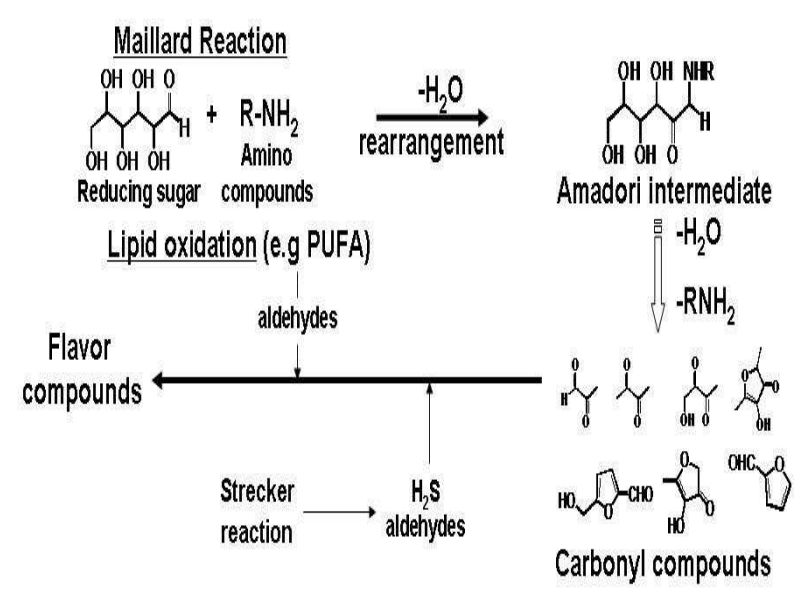

Figure 5. Interaction of the Maillard reaction and lipid oxidation for the production of aroma active compounds in miso prepared from lizard fish meat and soy bean.

The Maillard reaction between reducing sugar and free amino acids might play an important role in the development of several aroma-active compounds, including aldehydes, ketones, phenolic compounds, pyrazines, and furan derivatives, in miso during the advanced stages of maturation as shown in Fig.5. Furans and their derivatives can be a result of dehydrated or fermented condensates of carbohydrates either from koji or the nucleotide derivative ribose by Amadori rearrangement pathways [30,31]; however, they can also be produced as a result of oxidation of fatty acids such as 2-pentylfuran [32,33]. The presence of several furans and their derivatives such as furfural, which could be a result of glucose pyrolysis and the Maillard reaction, was confirmed in the fermented soybean paste [34]. Diacetyl formation in miso products is generally a result of glucose fermentation by the yeast through the EMP, where pyruvate and $\alpha$-acetolactate are produced as intermediates. Extracellular oxidative decarboxylation of $\alpha$-acetolactate ultimately yields diacetyl. 


\subsubsection{Catabolic Products of Fatty Acids}

Fish miso is a low-fat food, and ripened miso contains around 1\% lipids (wet mass) [2]; however, the small amount of fat in miso is important for the development of the typical flavor during the fermentation process. Flavoring compounds such as thiazoles, sulfur-containing compounds, esters, aldehydes, alcohols, and ketones are mostly present in the lipid phase of ripened fermented pastes [35]. In low-fat products like miso, there is an increased cross-linking within the curd/paste beginning in the early stage of maturation and persisting in the matured miso. It has been reported that cheddar cheese made from non-fat milk does not develop full aroma, even after 12 months [36]; however, substituting vegetable or even mineral oil for milk fat seems to favor a certain aroma development in cheddar cheese [37]. It is suggested that the fatty acid composition and natural emulsion of soybean/fish fat are important for flavor development. As in all types of fermented food, lipolytic (enzymatic hydrolysis by lipases and esterases) and oxidative (chemical) changes are likely to occur in miso. During ripening, hydrolysis is the principal biochemical transformation in miso, which leads to the production of free fatty acids (FFA), monoglycerides, diglycerides, and possibly glycerol. It has been reported that soybean lipids in sufu are also hydrolyzed to some extent into fatty acids. The added alcohol reacts with the fatty acids chemically or enzymatically to form esters, providing the pleasant odor of the product [38]. It is assumed that fish miso, being a low-fat product, can also be utilized by the cultures of koji by low-fat soybean miso, which was found to be helpful in enhancing flavor and overall acceptability.

The esters are produced during the alcoholic fermentation of the fish meat/soybean and koji, which is why the yeasts together with the fermentation condition play an important role in production. The esters of medium-chain fatty acids are of the most interest in this respect. Higher amounts of yeast strain and oxidative states of fermentation can accelerate the formation of this group of volatile compounds. Lipolytic enzymes are outstanding in terms of their ability to flavor the products (i.e., esters). A number of lipases have been reported for their ability to promote ester synthesis in low-water-content media such as those from Candida cylindracea, Pseudomonas fluorescens, Mucor miehei, Aspergillus spp., Rhizopus arrhizus, and C. rugosa [26]. Lipases are usually highly specific, which makes esterification between carboxylic acids and alcohols dependent on alcohol and/or acid chain length. Kumar, Modak, \&Madras [39] studied the esterification of fatty acids of different chain lengths using isoamyl alcohol with 3 different commercial lipases. Kwon, Hong, \& Yoon [40] investigated the ability of a number of lipases to catalyze enantiomeric selective synthesis of (S)-2-methylbutanoic acid methyl ester and found good results for lipases from Rhizomucor miehei, Aspergillusniger, and A. javanicus.

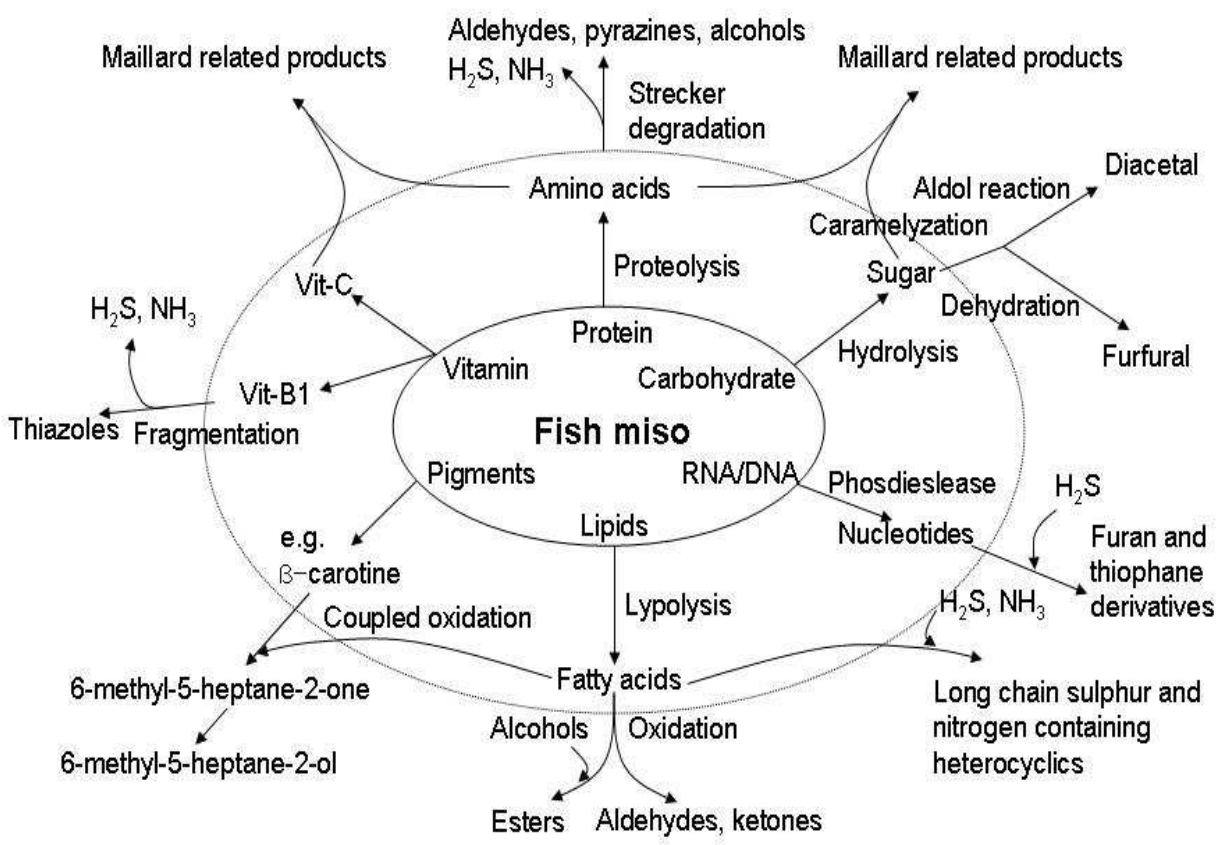

Figure 6. Flavor wheel of chemical reactions important to miso flavor.

The formation of aldehyde can be attributed to the decomposition of hydroperoxides and peroxyl radicals, which are supposed to be initial products of oxidized fat [41]. The volatile ketones were most likely the products of lipidand/or aminoacid degradation, and several have beenreported among the volatile compounds in natto fermentation rooms [42]. The auto-oxidation of fatty acids, particularly unsatu- rated ones (through hydroperoxides), has also been reported as a mechanism for the generation of methyl ketones [43]. Selke, Rohwedder, \& Dutton [44] reported the formation of a homologous series of methyl ketones as a result of $\beta$-oxidation (from the carbonyl end) ofthe carbon chain, followed by decarboxylation. Similarly, various other ketones could possibly be produced fromdistinct secondary 
degradation reactions involving diverse substances from the lipid fraction during fermentation and/or may be derived from the Maillard reaction. 6-Methyl-5-heptane-2-one might result as a coupled oxidation of fatty acids and pigments from the ingredients.

Formation of the major volatiles in miso products were suggested as a combined effect of fungal metabolism of amino acids, sugars, and lipids, as well as the Maillard reaction during the fermentation period, as presented in Fig.6.

\section{Conclusion}

Judging the relative abundances of the volatile constituents, we can conclude that miso prepared from soybeans and lizardfish meat is the result of alcoholic fermentation rather than acid fermentation; however, misoprepared from soybeans matured earlier (between 60 and 90 days) than that prepared from lizardfish meat (135 days). The stabilities in volatile lipid oxidation products, including certain aldehydes and ketones, indicate the oxidative stabilities of both miso products throughout the fermentation period. The compounds responsible for miso aroma were identified as 2methylpropanal, 3 - methylbutanal, 2, 4- nonadienal, 3methyl- 1-butanol, ethyl butanoate, ethyl isobutyrate, 2-methylethyl butanoate, 3-methylethyl butanoate, ethyl heptanoate, ethyl decanoate, 2,3-butanedione, dimethyl disulfide, dimethyl trisulfide, methional, 4-ethyl guaiacol, and 2-methyl butanoic acid. The major difference between miso prepared from lizardfish meatand that from soybeans was the relative abundance of those odor-active compounds that finally characterize the products. Metabolic capacity, substrate specificity of A. oryzae, and the Maillard reaction were presumed to determine the flavor profile of the end products of miso.

\section{References}

[1] Giri, K. Osako, and T. Ohshima, "Effect of raw materials on the extractive components and taste aspects of fermented. Fish paste - sakana miso," Fish. Sci., vol. 75, 2009, pp. 785-796.

[2] Giri, K. Osako, and T. Ohshima, "Extractive components and taste aspect of fermented fish pastes and bean pastes prepared using different koji molds as starters," Fish. Sci., vol. 75, 2009 , pp. 481-489.

[3] Giri, K. Osako, and T. Ohshima, "Identification and characterization of headspace volatiles of fish miso, a Japanese fish meat based fermented paste, with special emphasis on effect of fish species and meat washing," Food Chem., vol. 120, 2010, pp. 621-631.

[4] L. Liu, Y. M. Tzeng, "Water content and water activity for the production of cyclodepsipeptide in solid state fermentation," Biotechnol Lett, vol. 21, 1999, pp. 657-661.

[5] R. G. Berger, "Why novel biotechnology of aromas?," in Aroma Biotechnology, R. G. Berger, Ed.Berlin, Germany: Springer, 1995, pp. 35-41.
[6] G. Feron, P. Bonnarme, and A. Durand, "Prospects of the microbial production of food flavours," Trends Food Sci. Tech.,vol. 7, 1996, pp. 285-293.

[7] G. M. Pastore, Y. K. Park, and D. B. Min, "Production of a fruity aroma by Neurospora from beiju," Mycol. Res., vol. 98, 1994, pp. 25-35.

[8] E. Sugawara, S. Hashimoto, Y. Sakurai, and A. Kobayashi, "Formation by yeast of the HEMF (4-hydroxy-2 (or 5)-ethyl-5 (or 2)-methyl-3 (2H)-furanone) aroma components in Miso with aging," Biosci. Biotechnol. Biochem., vol. 58, 1994, pp. 1134-113.

[9] K. Ito, K. Yoshida, T. Ishikawa, and S. Kobayashi, "Volatile compounds produced by fungus Aspergillus oryzae in rice koji and their changes during cultivation," J. Ferment.Bioeng., vol. 70, 1990, pp. 169-172.

[10] M. L. Escamilla-Hurtado, S. E. Valdes-Martinez, J. Soriano-Santos, R. Gomez-Pliego, J. R. Verde-Calvo, A. Reyes-Dorantes, and A. Tomasini-Campocosio, "Effect of culture conditions on production of butter flavor compounds by Pediococcus pentosaceus and Lactobacillus acidophilus in semisolid maize-based cultures," Int. J. Food Microbiol., vol. 105, 2005, pp. 305-316.

[11] L. Janssens, H. L. de Pooter, E. J. Vandamme, and N. M. Schamp, "Production of flavours by microorganisms," Process Biochem., vol. 27, 1992, pp. 195-215.

[12] L. Marilley, and M. G. Casey, "Flavours of cheese products: metabolic pathways, analytical tools and identification of producing strains,” Int. J. Food Microbiol., vol. 90, 2004, pp. 139-159.

[13] R. A. Herbert and J. M. Shewan, "Roles played by bacterial and autolytic enzymes in the production of volatile sulphides in spoiling North Sea cod (Gadus morhua)," J. Sci. Food Agric., vol. 27, 1976, pp. 89-94.

[14] S. Landaud, S. Helinck, and P. Bonnarme, "Formation of volatile sulfer compounds and metabolism of methionine and other sulfur compounds in fermented food," Appl. Microbiol. Biotechnol., vol. 77, 2008, pp.1191-1205.

[15] S. Gummalla and J.R. Broadbent, "Tyrosine and phenylalanine catabolism by Lactobacillus cheese flavor adjuncts," J. Dairy Sci., vol. 84, 2001, pp. 1011-1019.

[16] F. Amarita, D. Fernandez-Espla, T. Requena, and C. Pelaez, "Conversion of methionine to methional by Lactococcus lactis," FEMS Microbiol. Lett., vol. 204, 2001, pp. 189-195.

[17] Girand, and T. Durance, "Headspace volatiles of sockeye and pink salmon as affected by retort process," J. Food Sci., vol. 65,2000, pp. 34-39.

[18] K. Dwivedi, "Meat flavor," CRC Crit. Rev. Food Technol, vol. 5,1975 , pp. 487-535.

[19] H. D. Belitz, and W. Grosch, "Food Chemistry", 1st Edn., Verlag, Heidelberg: Springer, vol. 87, Chapter 5, 1987, p.282.

[20] M. C. Montel, F. Masson, and R. Talon, "Bacterial role in flavour development," Meat Sci., vil. 49, 1998, pp. S111-S123.

[21] Z. Han, F. M. Rombouts, and M.J.R. Nout, "A Chinese fermented soybean food," Int. J. Food Microbiol., vol. 65, 2001, pp. 1-10. 
[22] M. L. Desjardins, D. Roy, and J. Goulet, "Growth of bifidobacteria and their enzyme profiles," J. Dairy Sci., vol. 73, 1990, pp. 299-307.

[23] Y. D. Hang and E. E. Woodams, "Apple pomace: A potential substrate for production of b-glucosidase by Aspergillus foetidus," Food Sci. Technol., vol. 27, 1994, pp. 587-589.

[24] A. Martino, P.G. Pifferi, and G. Spagna, "Production of b-glucosidase by Aspergillus niger using carbon sources derived from agricultural wastes," J. Chem Technol. Biotechnol., vol. 60, 1994, pp. 247-252.

[25] Gupta and D. Madamwar, "Solid-state fermentation of lignocellulosic waste for cellulase and b-glucosidase production by cocultivation of Aspergillus ellipticus and Aspergillus fumigatus," Biotechnol. Prog., vol. 13, 1997, pp. 166-169.

[26] M. A. Longo and M. A. Sanroman, "Production of Food Aroma Compounds," Food Technol. Biotechnol., vol. 44, 2006, pp. 335-353.

[27] E. Green, D. Herbert, and V. Subrahmanyan, "Carboxylase," J. Biol. Chem., vol. 138, 1941, pp. 327-339.

[28] H. Lehmann, G. Fischer, G. Heuber, K. D. Kohnert, and A. Schellenberger, "The influence of steric and electronic parameters on the substrate behaviour of a-oxo acids to yeast pyruvate decarboxylase," Eur. J. Biochem., vol. 32, 1973, pp. $83-87$.

[29] Kaminski, S. Stawicki, and E. Wasowicz, "Volatile flavor compounds produced by molds of Aspergillus, penicillium, and fungi imperfecti," Appl. Environ. Microbiol., vol. 27, 1974, pp. 1001-1004.

[30] R. L. Whistler and J. R. Daniel, "Carbohydrates," in Food Chemistry, O. Fennema, Ed. New York: Marcel Dekker Inc., 1985, pp. 69-137.

[31] M.A.J.S. van Boekel, "Formation of flavour compounds in the Maillard reaction," Biotechnol. Adv., vol. 24, 2006, pp. 230- 233 .

[32] J. Taylor and D. S. Mottram, "Composition and odour of volatiles from autoxidized methyl arachidonate" J. Sci. Food Agric., vol. 50, 1990, pp. 407-417.

[33] R. G. Krishnamurthy, T. H. Smouse, B. D. Mookherjee, B. R. Reddy, and S. S. Chang, "Identification of 2-pentyl furan in fats and oils and its relationship to the reversion flavor of soybean oil," J. Food Sci., vol. 32, 1967, pp. 372-374.

[34] S. M. Lee, B. C. Seo, and Y.-S. Kim, "Volatile compounds in fermented and acid hydrolyzed soy sauces," J. Food Sci., vol. 71, 2006, pp. 146-156.

[35] N. F. Olson, "The impact of lactic acid bacteria on cheese flavor," FEMS Microbiol. Lett., vol. 87, 1990, pp. 131-147.

[36] J. A. Ohren and S. L. Tuckey, Relation of flavour development in Cheddar cheese to chemical changes in fat of the cheese. J. Dairy Sci., vol. 52, 1969, pp. 598-607.

[37] A. Foda, E. G. Hammond, G. W. Reinbold, and D. K. Hotchkiss, "Role of fat in flavour of Cheddar cheese," J. Dairy Sci., vol. 57, 1974, pp. 1137-1142.

[38] C. Chou and C. H. Hwan, "Effect of ethanol on the hydrolysis of protein and lipid during the ageing of Chinese fermented soybean curd - Sufu," J. Sci. Food Agric., vol. 66, 1994, pp. 393-398.

[39] R. Kumar, J. Modak, and G. Madras, "Effect of chain length of the acid on the enzymatic synthesis of flavours in supercritical carbon dioxide," Biochem. Eng. J., vol. 23, 2005, pp. 199-202.

[40] D.Y. Kwon, Y.J. Hong, and S.H. Yoon, "Enantiomeric synthesis of (S)-2-methylbutanoic acid methyl ester, apple flavour, using lipases in organic solvent," J. Agric. Food Chem., vol. 48, 2000, pp. 524-530.

[41] J. D. Sink, "Lipid soluble components of meat, odor and their biochemical origin,” J. Am. Oil Chem. Soc., vol. 50, 1973, pp. 470-474.

[42] T. Tanaka, Z. Shoji, "Analysis of volatile compounds in the natto-fermenting room by gas chromatography-mass spectrometry," Nippon Shokuhin Kogyo Gakkaishi, vol. 40, 1993, pp. 656-660.

[43] P. Thomas, D. S. Dimick, and J. H. McNeil, "Sources of flavor in poultry skin," Food Technol., vol. 25, 1971, pp. 109-115.

[44] Selke, W. K. Rohwedder, and H. J. Dutton, "Volatile components from tristearin heated in air," J. Am. Oil Chem. Soc., vol. 52, 1975, pp. 232-235. 